\title{
Etude bioclimatique d'une futaie feuillue (Fagus silvatica L. et Quercus sessiliflora Salisb.) de l'Est de la France III. - Potentialités photosynthétiques des feuilles à différentes hauteurs dans le peuplement
}

\author{
M. DUCREY (*) \\ Station de Sylviculture et de Production \\ Centre national de Recherches forestières, I.N.R.A., \\ Champenoux, 54280 Seichamps
}

\begin{abstract}
Résumé
Pour étudier la photosynthèse nette dans une futaie feuillue, on a prélevé des rameaux de hêtre et de chêne à 10 hauteurs différentes dans le peuplement. Les courbes de photosynthèse nette en fonction de l'éclairement ont été établies en laboratoire grâce à une enceinte d'assimilation climatisée et régulée en température, en humidité et en gaz carbonique.

Pour le hêtre, la photosynthèse nette maximale par unité de surface foliaire augmente avec l'éclairement de croissance : elle est plus que doublée quand on passe des feuilles d'ombre $\left(4 \mathrm{mg} \mathrm{CO} . \mathrm{dm}^{-2} \mathrm{~h}^{-1}\right)$ aux feuilles de lumière $\left(9 \mathrm{mg} \mathrm{CO} \mathrm{dm}^{-2} \mathrm{~h}^{-1}\right)$. Dans le même temps, le poids spécifique des feuilles est multiplié par 3,5. Comme conséquence, la photosynthèse par unité de matière sèche diminue quand l'éclairement de croissance augmente : elle est $50 \mathrm{p} .100$ plus élevée à l'ombre $\left(14 \mathrm{mg} \mathrm{CO}_{2 .} \mathrm{g}^{-1} \mathrm{~h}^{-1}\right)$ qu'à la lumière $\left(9 \mathrm{mg} \mathrm{CO} \mathrm{gg}^{-1} \mathrm{~h}^{-1}\right.$ ).

Pour le chêne des tendances analogues sont observées.

Ces résultats sont discutés en fonction des caractéristiques morphologiques, anatomiques et physiologiques des feuilles et par comparaison avec les données bibliographiques. La photosynthèse par unité de volume foliaire apparaît comme un critère stable indépendant de l'éclairement de croissance des différentes feuilles.
\end{abstract}

\section{1. - Introduction}

La croissance de la forêt est la conséquence de la production photosynthétique primaire qui a lieu à tous les niveaux du couvert forestier. La photosynthèse n'est évidemment pas le seul processus physiologique qui conditionne la croissance des

(*) Adresse actuelle : Station de Bioclimatologie, I.N.R.A. Antilles-Guyane, 97170 Petit-Bourg, Guadeloupe (F.W.I.). 
arbres et de la forêt mais c'est le processus primaire par quoi tout commence. Aussi la connaissance du fonctionnement photosynthétique est-elle fondamentale pour l'étude de la croissance des arbres et des peuplements forestiers.

C'est pourquoi différents chercheurs se sont penchés sur ce problème et parmi eux : Schulze \& Koch (1971), Starzecki (1974), Künstle \& Mitscherlich (1975). Mais les résultats obtenus sont très hétérogènes et parfois même contradictoires, si bien qu'il est actuellement très difficile de faire une synthèse des différents processus physiologiques qui interviennent dans le fonctionnement photosynthétique des couverts forestiers. Le débat est donc encore largement ouvert et c'est pour cela que nous avons entrepris à Nancy des études dans ce domaine.

Dans un premier article (Aussenac \& DuCREY, 1977) nous avons étudié les différents profils microclimatiques ainsi que les caractéristiques anatomiques et morphologiques de l'appareil foliaire dans une futaie mélangée de hêtre et de chêne. Ceci nous a amenés à considérer deux zones bien caractéristiques.

Dans la partie ensoleillée des cimes, les températures diurnes sont élevées et l'humidité faible, ce qui, en dépit des apports énergétiques importants, peut créer des conditions de photosynthèse défavorables en raison de l'éventualité d'une fermeture plus ou moins forte des stomates.

Dans la partie où les couronnes deviennent jointives et où les feuilles sont déjà ombragées, la température diminue et l'humidité devient plus élevée. Les apports énergétiques sont encore suffisants et on peut penser que c'est à ce niveau que les processus photosynthétiques sont les plus actifs.

L'étude des caractéristiques anatomiques et morphologiques de l'appareil foliaire a montré les relations croissantes qui existent entre l'éclairement relatif dans lequel vivent les feuilles d'une part, le poids spécifique et l'épaisseur des feuilles d'autre part. Elle a permis de mettre en évidence l'adaptation de l'appareil foliaire aux différentes conditions énergétiques.

Ce premier travail a été complété par l'étude de la photosynthèse nette qui fait l'objet du présent article. Il est aussi à noter que dans le cadre de l'analyse bioclimatique de cette futaie feuillue de hêtre et de chêne, des études ont été entreprises sur le bilan hydrique et l'évapotranspiration réelle au niveau du peuplement (AussenAC \& Granier, 1979).

\section{2. - Matériel et méthode d'étude}

\section{1. - Parcelle et dispositif expérimentaux}

Le dispositif expérimental a été installé dans la parcelle 26 de la forêt domaniale d'Amance (Meurthe-et-Moselle) à une quinzaine de kilomètres à l'Est de Nancy.

Le peuplement est constitué d'une futaie de hêtre (Fagus silvatica L.) à couvert fermé et avec un sous-étage très réduit. On rencontre aussi en mélange des chênes (Quercus sessiliflora Salisb.), quelques tilleuls (Tilia cordata Mill.), charmes (Carpinus betulus L.) et frênes (Fraxinus excelsior L.). Le peuplement, issu d'une régénération 
naturelle, a un âge moyen de 80 ans, une hauteur moyenne de 23 mètres et une densité de 744 arbres à l'hectare. Une description plus précise du peuplement est donnée dans un article précédent (Aussenac \& Ducrey, 1977).

Sur une tour métallique de 25 mètres de hauteur construite au sein du peuplement, 10 points de mesure ont été choisis pour établir les profils microclimatiques. C'est à proximité de chacun de ces 10 points qu'ont été prélevés les rameaux de hêtre et de chêne utilisés pour les études de photosynthèse.

\section{2. - Dispositif d'étude de la photosynthèse nette}

Des rameaux de grande taille contenant entre 50 et 100 feuilles sont prélevés dans le peuplement et immédiatement immergés dans un récipient plein d'eau. Dans la minute qui suit, ils sont coupés sous l'eau à la taille convenable. Ils sont alors prêts à être transportés au laboratoire et à être installés dans l'enceinte d'assimilation. Les rameaux de hêtre ont entre 5 et 10 feuilles ce qui représente une surface moyenne de 200 à $250 \mathrm{~cm}^{2}$ tandis que les rameaux de chêne ont entre 3 et 5 feuilles pour une surface foliaire de 350 à $400 \mathrm{~cm}^{2}$.

L'utilisation de cette méthode dite «du rameau coupé» fait souvent l'objet de critiques et on lui reproche de modifier la réponse du végétal. C'est ainsi que Mousseau (1968) a trouvé que le fait d'utiliser des feuilles coupées, dans le cas d'une plante herbacée, avait pour effet d'augmenter la photosynthèse des plants de lumière et de diminuer celle des plants d'ombre. Nous n'avons quant à nous trouvé aucune différence entre la photosynthèse du plant entier et celle du rameau coupé dans le cas de jeunes peupliers cultivés en pleine lumière et bien pourvus en eau. Il est difficile de trouver une explication précise à ces différentes observations mais nous pensons que le fait de maintenir dans l'eau un rameau coupé, a pour effet de supprimer une partic des résistances au transfert de l'cau dans la plante et donc d'obtenir une photosynthèse maximale. Il s'ajoute à cela le fait que chez une plante ligneuse, la tige est un tampon plus important que chez les plantes herbacées d'où l'absence de différences.

Nous admettons donc dans nos expériences que les rameaux photosynthétisent au maximum de leurs potentialités ce qui n'est pas forcément le cas dans les conditions naturelles.

Le dispositif d'étude de la photosynthèse a fait par ailleurs l'objet d'une description détaillée (DuCrey, 1979) et est représenté schématiquement par la figure 1.

L'enceinte d'assimilation, fabriquée par Siemens, est construite en plexiglas et a un volume utile de $38 \mathrm{~cm} \times 21 \mathrm{~cm} \times 7 \mathrm{~cm}$. La régulation de la température est assurée au moyen de blocs Peltier et de sondes à résistance de platine. Le brassage interne de l'air assure une bonne régulation et la puissance des blocs Peltier permet d'atteindre des températures de lordre de $\pm 20^{\circ} \mathrm{C}$ par rapport à la température ambiante. L'humidité est maintenue constante à l'entrée de l'enceinte et est régulée grâce à un système de piège à eau à point de rosée variable ct de sondes à chlorure de lithium installées sur un circuit en dérivation. La teneur en gaz carbonique est régulée par un dispositif original qui fonctionne d'après les indications d'un analyseur absolu de $\mathrm{CO}_{2}$ installé à la sortie de l'enceinte. L'éclairage du dispositif est assuré par des lampes à vapeur de mercure (Mazda, MAF 400 et MAF 2000) qui permettent de faire varier le rayonnement global de 0 à $400 \mathrm{~W} \mathrm{~m}^{-2}$. La photosynthèse nette est 
calculée grâce à un analyseur différentiel à infra-rouge qui mesure la différence de concentration en $\mathrm{CO}_{2}$ de l'air à l'entrée et à la sortie de la chambre d'assimilation.

Pour cette expérience, la température interne était fixée à $20^{\circ} \mathrm{C}$, l'humidité relative à $70 \mathrm{p}$. 100 . Un ventilateur interne assure au voisinage des feuilles une ventilation d'environ $1 \mathrm{~m} \mathrm{~s}^{-1}$.

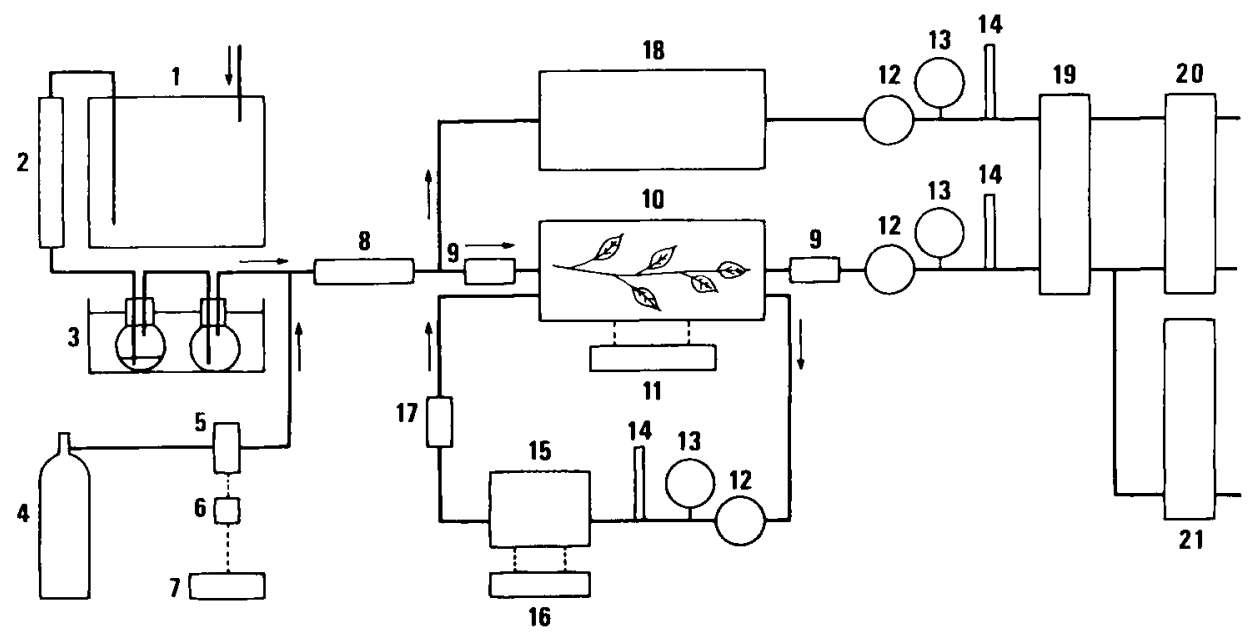

FIG. 1

Schéma général du dispositif d'étude de la photosynthèse

1 : récipient mélangeur; 2 : tube à chaux sodée; $3:$ bain thermostaté $; 4:$ gaz sous pression contenant 90 p. 100 de $\mathrm{N}_{2}$ et 10 p. 100 de $\mathrm{CO}_{2} ; 5$ : vanne; 6 : servomoteur ; 7 : système de régulation de la teneur en $\mathrm{CO}_{2} ; 8$ : filtre à poussières ; 9 : sonde d'humidité à chlorure de lithium ; 10 : enceinte d'assimilation ; 11 : système de régulation de la température ; 12 : pompe; 13 : manomètre; 14 : débitmètre; 15 : piège à eau à point de rosée variable pour la régulation de l'humidité ; $16:$ système de régulation de l'humidité ; 17 : sonde d'humidité du circuit en dérivation ; 18 : récipient de compensation du circuit de référence ; 19 : piège à eau réglé à $1{ }^{\prime \prime} \mathrm{C} ; 20$ : analyseur différentiel de $\mathrm{CO} ; 21$ : analyseur absolu de $\mathrm{CO}_{2}$.

Block diagram for the gas-exchange chamber used for photosynthesis measurements

1 : mixing vessel; 2 : soda lime column; 3 : temperature controlled water bath ; 4 : high pressure gas with 90 p. $100 \mathrm{~N}_{\text {: }}$ and 10 p. $100 \mathrm{CO}_{2} ; 5$ : gas valve ; 6 : servomotor;

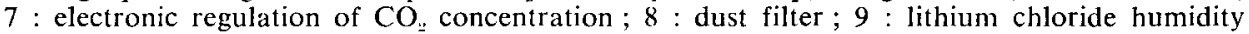
sensor ; 10 : gas-exchange chamber ; 11 : temperature regulator ; $12:$ pump ; 13 : differential pressure manometer ; 14 : flowmeter (rotameter) ; 15 : water trap with variable dew point; 16 : humidity regulator; 17 : lithium chloride humidity sensor of the by-pass circuit; 18: compensation vessel ; 19 : water trap with constant dew-point of $1{ }^{\circ} \mathrm{C} ; 20$ : differential infra-red gas analyser ; 21 : absolute gas analyser.

\section{3. - Résultats}

Les courbes de photosynthèse nette en fonction de l'éclairement ont été construites à partir des mesures d'échanges gazeux effectuées à 5 éclairements différents : 25,45 , 
100,180 et $380 \mathrm{~W} \mathrm{~m}^{-2}$. Ces mesures de photosynthèse nette ont été faites en laboratoire avec des rameaux prélevés à 9 niveaux dans le peuplement pour le hêtre, et à 4 niveaux seulement pour le chêne.

Les 10 niveaux de référence choisis dans cette hêtraie sont numérotés de $\mathbf{I}$ à $\mathbf{X}$ du sommet du peuplement jusqu'au sol (Aussenac \& Ducrey, 1977). Ainsi les rameaux prélevés au niveau II ont poussé pratiquement en pleine lumière tandis que ceux prélevés aux niveaux VIII, IX et $\mathrm{X}$ correspondent au sous-étage et ont poussé sous des éclairements relatifs inférieurs à 10 p. 100.

Il n'a pas été possible de faire des répétitions en étudiant plusieurs rameaux à chaque niveau mais les mesures ont été faites pendant deux années consécutives, du 18 au 28 juin 1974 et du 28 juillet au 7 août 1975 , et les résultats diffèrent peu d'une année à l'autre. La figure 2 montre les résultats obtenus en 1974 pour le hêtre et le chêne. Dans tous ces résultats, nous n'avons pas tenu compte de la respiration des tiges. Il nous a paru possible de négliger ce facteur, qui a normalement pour effet de sous-estimer la photosynthèse nette, en raison de la très petite longueur de tige effectivement présente dans l'enceinte par rapport à la surface foliaire des rameaux.

Pour le hêtre, les rameaux supérieurs (niveau II) ont une photosynthèse nette maximale atteignant presque $9 \mathrm{mg} \mathrm{CO}_{2}$ par $\mathrm{dm}^{2}$ de surface foliaire et par heure tandis que les rameaux de sous-étage (niveaux VIII, IX et X) ont une photosynthèse nette maximale voisine de $4 \mathrm{mg} \mathrm{CO}_{2} \mathrm{dm}^{-2} \mathbf{h}^{-1}$; l'activité des feuilles du haut est plus de 2 fois supérieure à celle des feuilles du bas. Elles arrivent à saturation pour des éclairements de $180 \mathrm{~W} \mathrm{~m}^{-2}$ tandis que celles du bas sont saturées entre 50 et $100 \mathrm{~W} \mathrm{~m}^{-2}$.

Pour le chêne, on constate tout d'abord que le palier de saturation n'a pas été atteint même à $380 \mathrm{~W} \mathrm{~m}^{-2}$. On trouve ensuite des phénomènes analogues à ceux indiqués pour le hêtre, mais moins tranchés car l'arbre étudié est un chêne dominé, isolé parmi les hêtres et ayant un houppier très peu développé.

Dans ce qui précède, la photosynthèse a été rapportée à l'unité de surface foliaire. Mais si on la calcule par rapport à la matière sèche foliaire on obtient des résultats sensiblement différents (tableau 1). Pour le hêtre, on observe une tendance nette de la photosynthèse par unité de matière sèche à augmenter lorsque l'éclairement relatif diminue. La photosynthèse des feuilles d'ombre est alors 50 p. 100 plus forte que celle des feuilles de lumière. On observe la même tendance chez le chêne mais les résultats ne sont pas suffisamment nombreux pour montrer parfaitement cette variation.

La comparaison entre le chêne et le hêtre montre que, en pleine lumière, les potentialités photosynthétiques du chêne, qu'elles soient exprimées par rapport à la surface foliaire ou à la matière sèche, sont inférieures à celles du hêtre. Ceci se retrouve pour les feuilles situées à l'ombre, la photosynthèse des rameaux prélevés à 7,4 p. 100 d'éclairement étant de $5,16 \mathrm{mg} \mathrm{CO}_{2} \mathrm{dm}^{-2} \mathrm{~h}^{-1}$ pour le hêtre et de $2,76 \mathrm{mg} \mathrm{CO} \mathrm{Cm}^{-2} \mathrm{~h}^{-1}$ pour le chêne. Ceci, ajouté au fait qu'on ne trouve plus de feuilles de chêne au-dessous du niveau VII (à $12,60 \mathrm{~m}$ au-dessus du sol) tandis qu'on trouve sur le sol de jeunes régénérations de hêtre, traduit une très mauvaise adaptation du chêne à l'ombrage alors que le hêtre semble parfaitement adapté à ces conditions. 


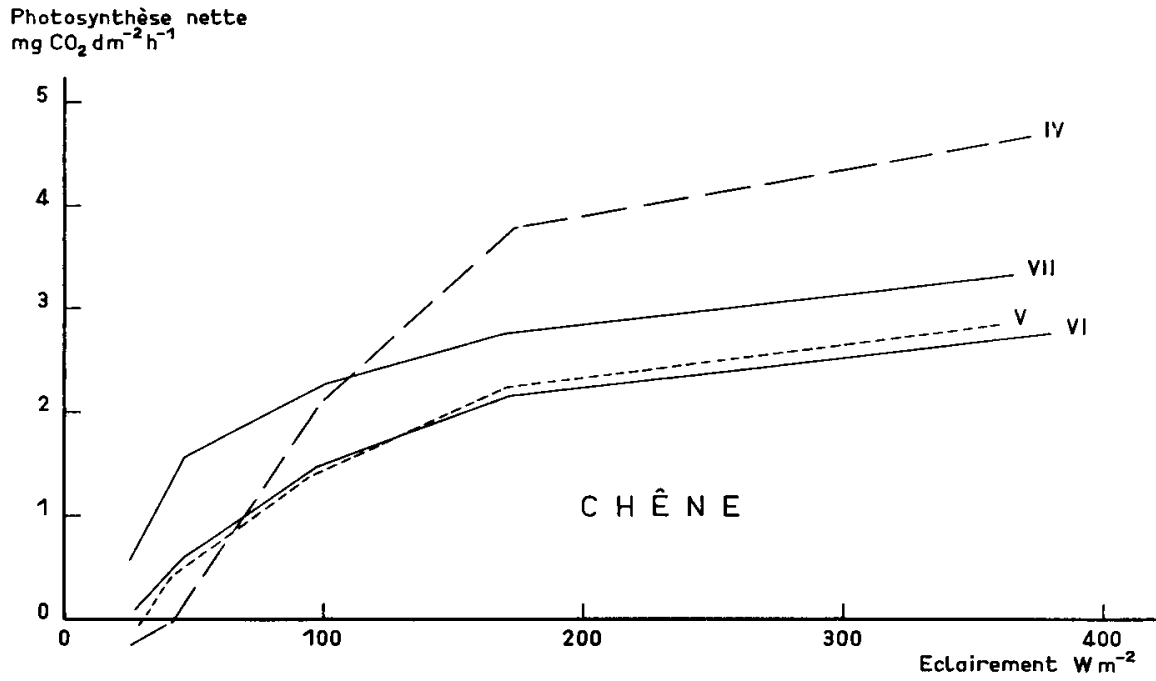

Photosynthese nette $\mathrm{mg} \mathrm{CO} 2 \mathrm{dm}^{-2} \mathrm{~h}^{-1}$

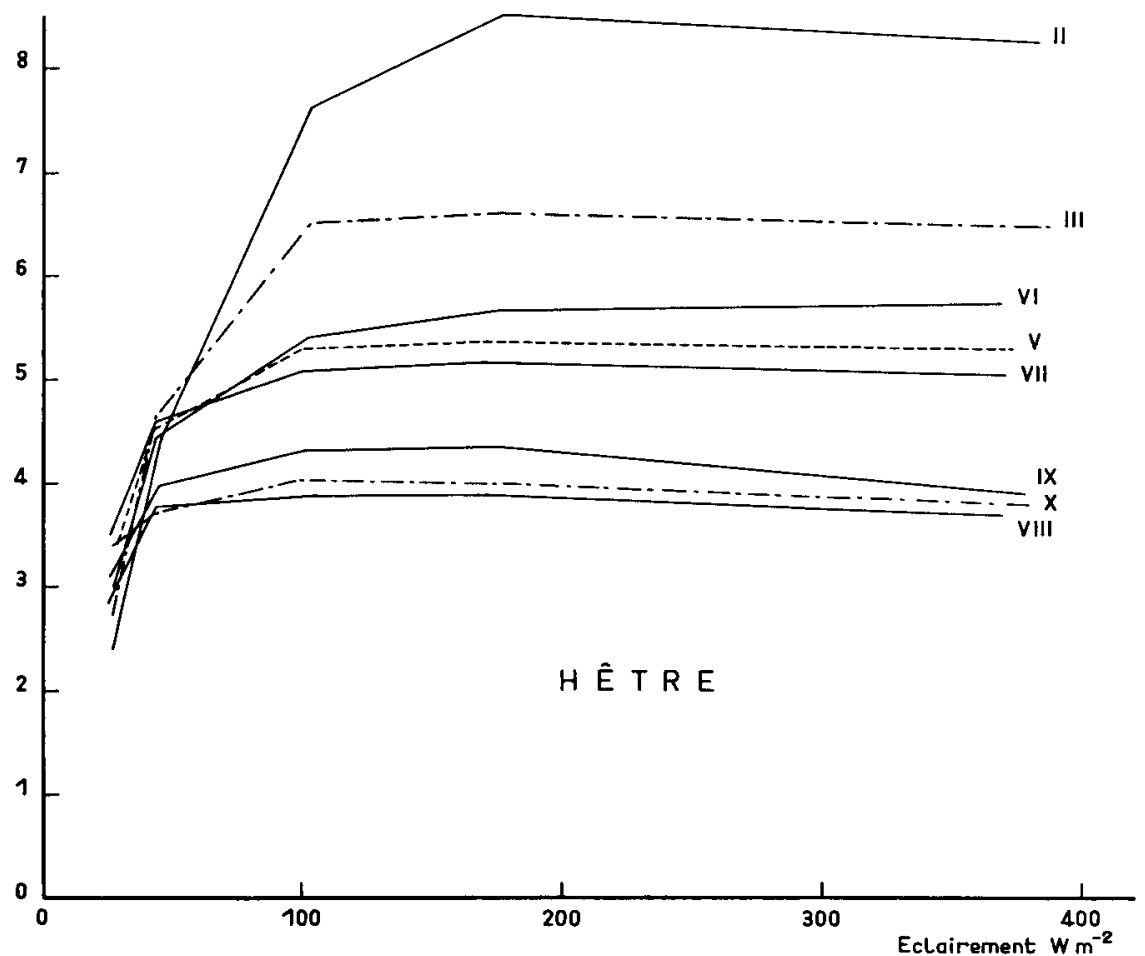

FIG. 2

Courbes de photosynthèse nette en fonction de l'éclairement pour des rameaux de hêtre et de chêne prélevés à différents niveaux dans le peuplement (18-28 juin 1974)

Le niveau II correspond à la cime des arbres: feuilles de pleine lumière, tandis que le niveau $X$, au voisinage du sol, est caractéristique des feuilles d'ombre.

Light curves of net photosynthesis per leaf area unit of beech and oak twigs took at various levels in the tree crowns (june 18-28, 1974) 


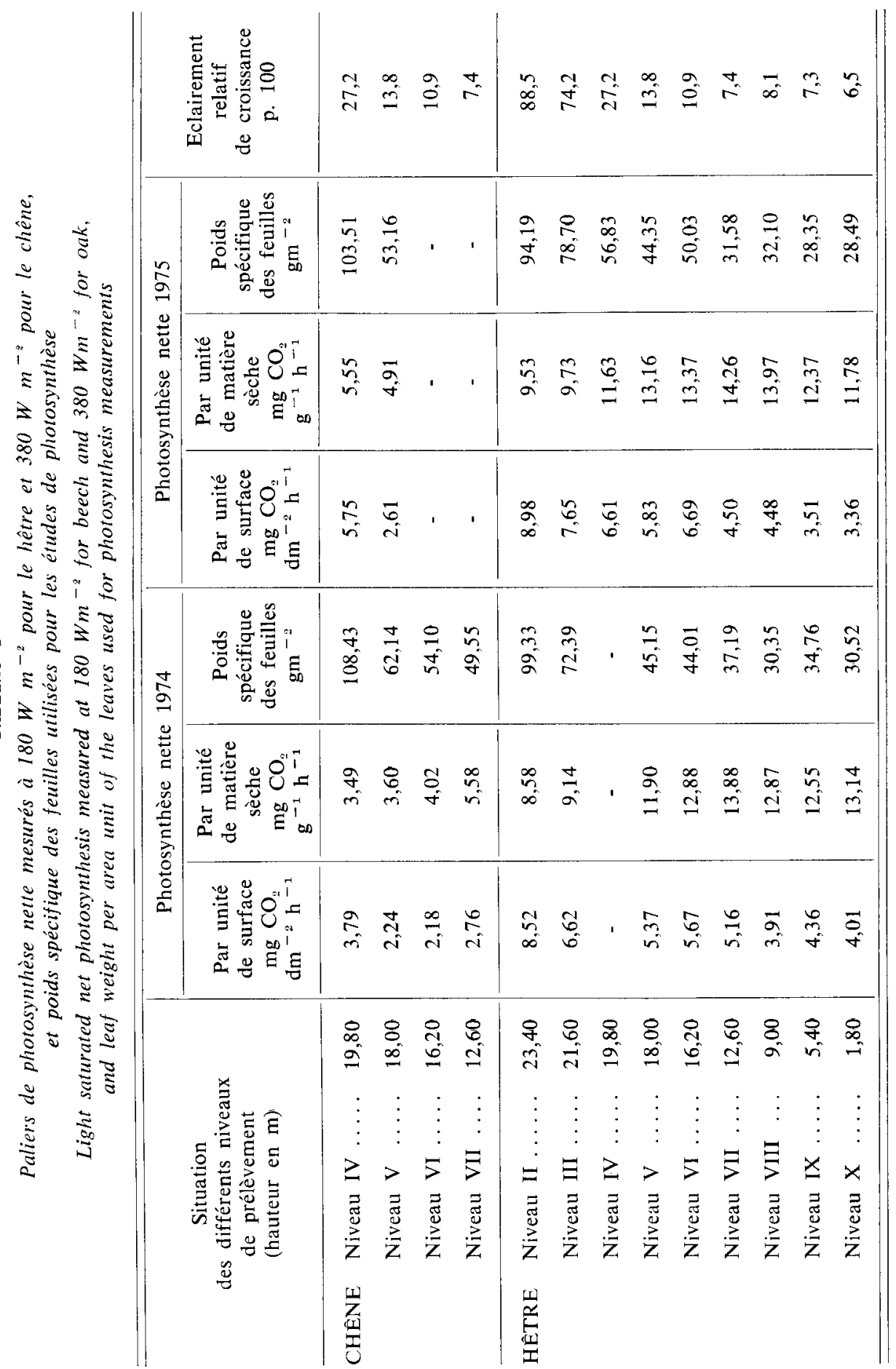


Pour mieux expliquer le gradient vertical au sein du peuplement, nous avons essayé de relier la photosynthèse nette maximale à chaque niveau, exprimée par unité de surface foliaire, aux paramètres microclimatiques et anatomiques les plus caractéristiques de ces niveaux.

Sur la figure 3 , on a reporté le palier de saturation des différentes courbes de photosynthèse du hêtre en fonction d'une part de l'éclairement relatif et d'autre part du poids spécifique des feuilles. On trouve une relation croissante entre la photosynthèse et le poids spécifique des feuilles. Quand on passe des feuilles d'ombre aux feuilles de lumière la photosynthèse est un peu plus que doublée tandis que le poids spécifique des feuilles est multiplié approximativement par 3,5.

On constate aussi avec l'éclairement relatif une relation croissante qui peut être divisée en deux parties : une première partie correspondant à des éclairements relatifs inférieurs à 15 p. 100 et pour laquelle le palier de photosynthèse augmente très rapidement avec l'éclairement relatif, et une deuxième partie pour laquelle la photosynthèse est beaucoup moins influencée par l'éclairement relatif. La première partie de la courbe correspond aux niveaux $\mathrm{V}$ à $\mathrm{X}$ qui sont situés dans la partie des houppiers constamment à l'ombre tandis que les niveaux II, III et IV, pour lesquels la photosynthèse est moins dépendante de l'éclairement, sont situés dans la zone éclairée des houppiers.

La figure 3 montre aussi les variations de la photosynthèse nette maximale exprimée par unité de matière sèche en fonction d'une part de l'éclairement relatif et d'autre part du poids spécifique des feuilles. La photosynthèse décroît de manière quasi linéaire quand l'éclairement relatif ou le poids spécifique des feuilles augmente. Quand on passe de la lumière à l'ombre la photosynthèse augmente de 50 p. 100 tandis que dans le même temps le poids spécifique des feuilles est divisé par 3,5.

Pour le chêne nous ne disposons pas de suffisamment de données pour établir de telles relations.

\section{4. - Discussion}

Les courbes de photosynthèse que nous avons construites montrent les différences qui existent entre les feuilles d'ombre et les feuilles de lumière, mais aussi un gradient continu de variation entre ces deux extrêmes. Ceci constitue un premier type d'adaptation à la lumière des différentes feuilles situées sur le même arbre et soumises par ombrage mutuel à des conditions lumineuses très différentes. Un second type d'adaptation à la lumière concerne des plants différents de la même espèce, par exemple des jeunes plants de hêtre ayant poussé les uns dans le sous-bois, les autres en clairière ou en plein découvert. Un dernier type d'adaptation, enfin, se situe au niveau des espèces : utilisation différente de la lumière pour le chêne, essence de lumière et le hêtre, essence d'ombre.

Mais quelque soit le cas envisagé, l'adaptation à la lumière ou à l'ombrage se traduit toujours à peu près de la même manière, par des modifications plus ou moins importantes de l'appareil foliaire. 

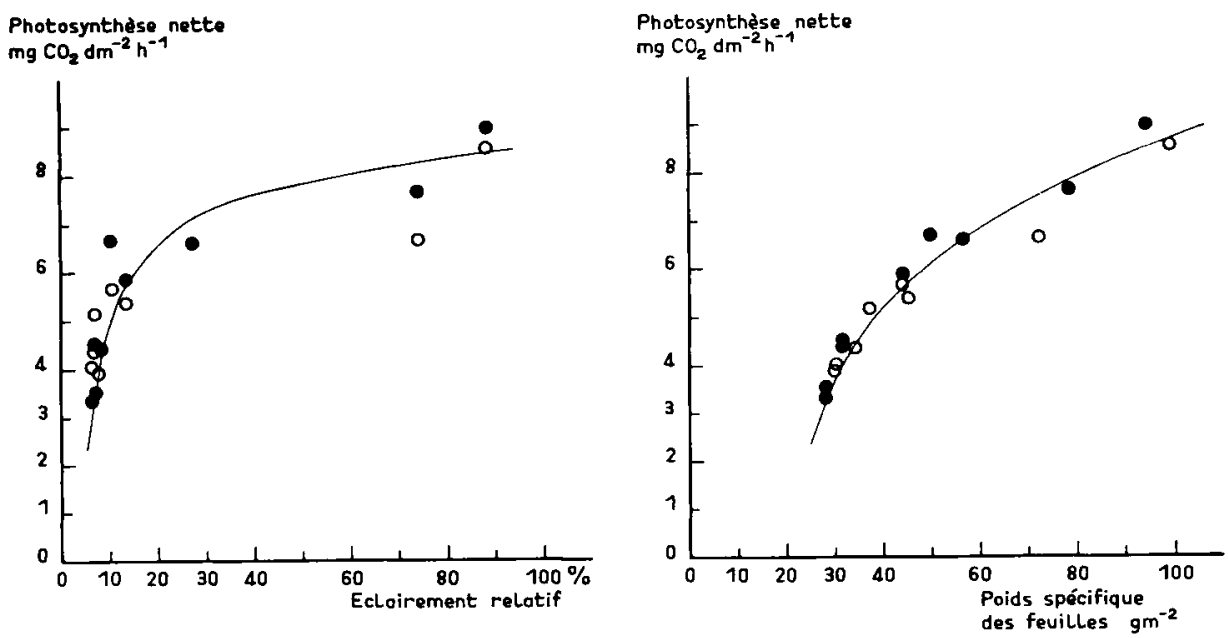

Photosynthèse nette
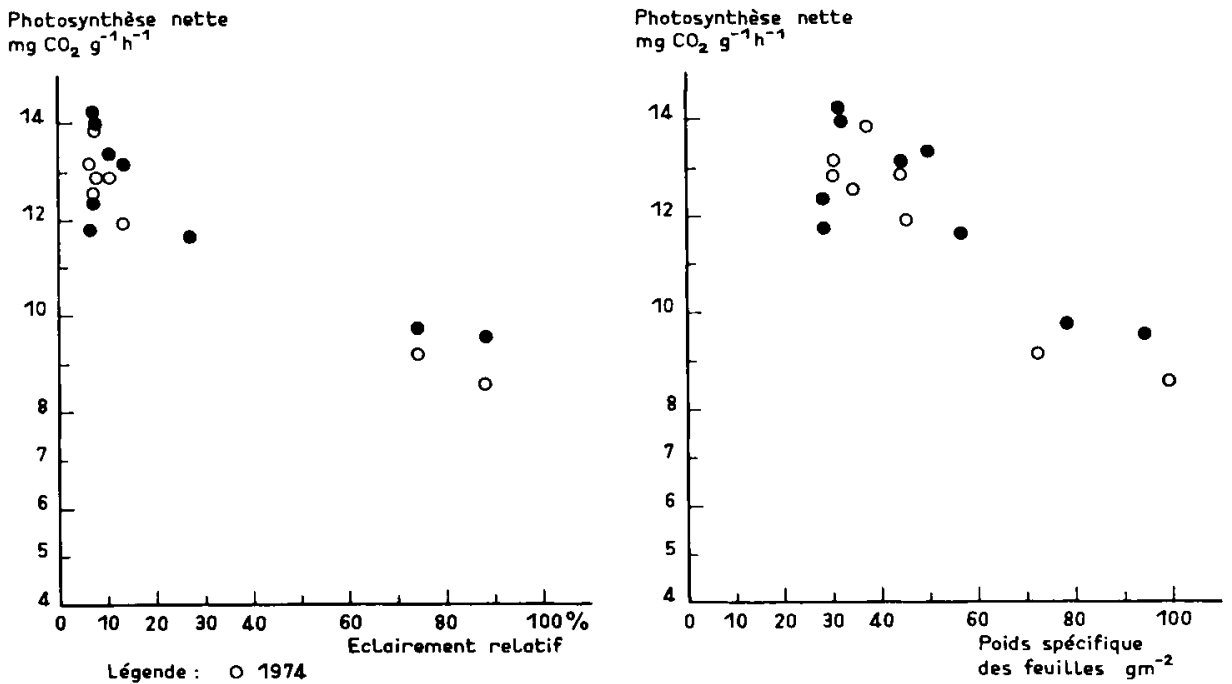

FIG. 3

Relations chez. le hêtre entre la photosynthèse nette, l'éclairement relatif de croissance et le poids spécifique des feuilles

- En ordonnées, on a porté en haut la photosynthèse nette par unité de surface et en bas la photosynthèse nette par unité de matière sèche.

- En abscisses, on a à gauche l'éclairement relatif de croissance et à droite le poids spécifique des feuilles $(O: 1974$, $\bullet: 1975)$.

Relations between maximum rate of net photosynthesis expressed as dry-matter weight units and leaf area units on the one hand, relative daylight intensity of growth and specific weight of leaves on the other hand 


\section{1. - Photosynthèse et caractéristiques foliaires}

Nous avons vu que la photosynthèse par unité de surface augmente du simple au double quand on passe des feuilles d'ombre aux feuilles de lumière et que, dans les mêmes conditions, la photosynthèse par unité de matière sèche reste à peu près constante et même qu'elle a tendance à diminuer. Ces résultats sont conformes à ceux des auteurs qui ont étudié les problèmes d'adaptation à la lumière chez les plantes herbacées, par exemple Bü̈rkman \& Holmgren (1963) et Mousseau (1969, 1977), ainsi qu’à ceux des chercheurs qui se sont occupés des arbres forestiers.

C'est ainsi que Schulze (1970) trouve pour le hêtre que la photosynthèse par unité de surface est approximativement 2 fois plus forte pour les feuilles de lumière que pour les feuilles d'ombre. Il ne faut bien entendu retenir que l'ordre de grandeur car les variations que l'on observe sont dues à des différences dans l'éclairement de croissance des feuilles d'ombre qui peut être plus ou moins faible selon la densité du peuplement. StaRzecki (1975) qui a étudié Acer pseudoplatanus, Fraxinus excelsior et Tilia cordata trouve des résultats analogues, les paliers de photosynthèse nette étant selon les espèces entre 2 et 4 fois plus élevés pour les feuilles de lumière que pour les feuilles d'ombre. Pour le chêne (Quercus robur L.), Penka et al. (1971) ont étudié la photosynthèse par la méthode gravimétrique en prenant des échantillons au sommet, au milieu et au bas de la couronne dans un peuplement adulte. Ils trouvent que la quantité de produits élaborés diminue du haut vers le bas quand on la rapporte à l'unité de surface et qu'elle augmente quand on la rapporte à l'unité de matière sèche. Nos résultats se rapprochent de ceux de Penka et al. Les autres auteurs cités précédemment ont trouvé, quant à eux, que la photosynthèse par unité de matière sèche reste à peu près constante tout au long du gradient d'éclairement depuis les feuilles de lumière jusqu'aux feuilles d'ombre.

Les explications de ces différentes observations doivent être recherchées dans les caractéristiques anatomiques et morphologiques de l'appareil foliaire, siège des réactions photosynthétiques.

En première approche, elles sont la conséquence directe des variations du poids spécifique des feuilles. En effet le tableau 1 montre que la photosynthèse par unité de surface double tandis que le poids spécifique des feuilles est un peu plus que triplé. Il en résulte que dans le même temps la photosynthèse par unité de matière sèche décrô̂t de près de 50 p. 100.

Si de plus on rapporte la photosynthèse à l'unité de volume de feuille - des résultats antérieurs (AUSSENAC \& DUCREY, 1977) montrent que l'épaisseur des feuilles varie du simple au double entre le niveau du sol et le sommet du peuplement - on voit qu'elle reste constante pour toutes les feuilles que celles-ci soient à l'ombre ou au soleil.

STARzecki (1974) a tenté d'expliquer les variations de la photosynthèse et notamment la forme de la relation entre la photosynthèse et l'éclairement relatif (figure 3) en étudiant l'anatomie des feuilles et plus particulièrement le tissu palissadique. Il a trouvé que lorsque la lumière diminue, l'épaisseur du tissu palissadique - caractéristique des feuilles de lumière - diminue aussi, tandis que le parenchyme lacuneux - présent à la fois chez les feuilles d'ombre et de lumière - n'est pas modifié. Ceci lui permet de conclure qu'une diminution de l'éclairement relatif jusqu'à 30 p. 100 modifie peu la photosynthèse en dépit d'une réduction importante de l'épaisseur des 
feuilles - ce que nous avons aussi constaté, mais au-dessous de 15 p. 100 - et que le parenchyme palissadique a une aetivité photosynthétique moindre que celle du parenchyme lacuneux. Nous ne pouvons toutefois souscrire entièrement à cette hypothèse car nous avons constaté (AUSSEnaC \& DUCREy, 1977) que le rapport entre l'épaisseur du tissu palissadique et celle de la fcuille cntière restait constant du sommet au bas des couronnes et était de 0,43 pour le chêne et de 0,40 pour le hêtre.

Il est aussi possible de rapporter la photosynthèse à la quantité de chlorophylle et dans ce cas, Schulze (1970) trouve que la photosynthèse des feuilles de lumière est, chez le hêtre, 3 fois supérieure à celle des feuilles d'ombre. Il cn résulte que la quantité de chlorophylle par unité de surface foliaire a tendance à diminuer quand on passe de l'ombre à la lumière. C'est aussi ce que trouvent Wassink et al. (1956) et Boardman (1977). Ce dernier constate aussi que chez les feuilles d'ombre, l'accroissement de la taille des chloroplastes et de la teneur en chlorophylle par chloroplaste est loin de compenser la diminution du nombre de chloroplastes par unité de surface foliaire, et c'est pourquoi quand on rapporte la photosynthèse à la quantité de chlorophylle elle augmente plus entre l'ombre et la lumière que si on la rapportait à la surface foliaire.

Ces résultats sont en quelque sorte confirmés par l'analyse enzymatique car on trouve de manière générale (BOARDMAN, 1977) qu'une plus grande capaoité à la fixation du $\mathrm{CO}_{2}$ par unité de surface est associée à une plus grande quantité d'enzymes de carboxylation.

En fin de compte, si la photosynthèse par unité de surface foliaire est autant affectée par l'éclairement de croissance, c'est parce que la feuille subit elle-même des transformations morphologiques et anatomiques importantes. Mais si on se réfère à des données telles que la matière sèche ou encore mieux au volume de la feuille, il apparaît que la photosynthèse est alors peu affectée par l'éclairement de croissance.

\section{2. - Production photosynthétique d'un peuplement}

Dans la discussion qui précède, on a comparé entre eux des plants entiers ayant été élevés dans des conditions d'ombre ou de pleine lumière ainsi que des rameaux détachés de l'arbre sur lequel ils ont poussé soit à l'ombre, soit à la lumière. D'autres études ont pu être faites vraiment en conditions naturelles dans des enceintes climatisées de même type que la nôtre mais sur des rameaux rattachés à l'arbre. C'est le cas notamment de Schulze \& Koch (1971) et de KüNSTl.e \& Mitscherl.ich (1975, 1977). Malheureusement leurs résultats sont assez divergents et difficiles à interpréter.

Schulze \& Koch trouvent, pour l'ensemble d'une saison de végétation, que la quantité de $\mathrm{CO}_{3}$, assimilée par unité de matière sèche par les feuilles de lumière est à peine 6 p. 100 supérieure à celle assimilée par les feuilles d'ombre bien que le fonctionnement dans le temps de ces 2 types de feuilles ne soit pas le même. En effet, au printemps les feuilles de lumière photosynthétisent plus rapidement et lcur production est plus élevée. Le phénomène s"inverse à la fin de juillet et les feuilles d’ombre deviennent aiors prépondérantes. 


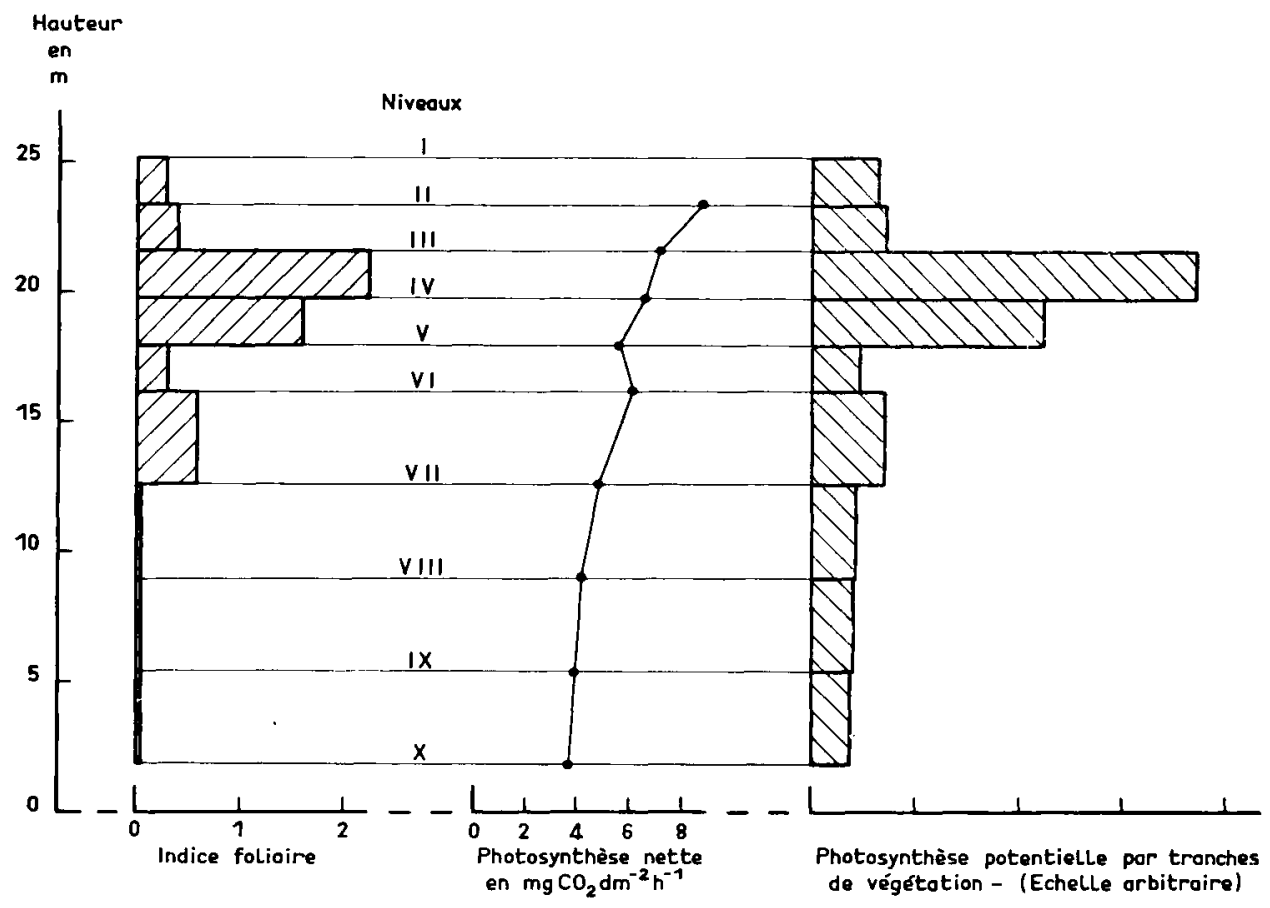

Fig. 4

Potentialités photosynthétiques d'un peuplement de hêtre par tranches horizontales de végétation

Ce graphique est obtenu en combinant le profil d'indice foliaire (L.A.I.) de ce peuplement, à gauche, et les valeurs de photosynthèse nette maximale pour chaque niveau, au milieı.

Potential photosynthesis rates at various levels in a beech stand

This graphic is a combination between leaf area index and light-saturated net photosynthesis for each level.

Künstle \& Mitscherlich, ayant étudié le douglas, le hêtre, le bouleau et le pin sylvestre, trouvent que la photosynthèse par unité de matière sèche est toujours plus faible pour les feuilles d'ombre que pour les feuilles de lumière. Pour l'ensemble d'une saison de végétation, la photosynthèse des feuilles d'ombre ne représente par rapport à celle des feuilles de lumière que 20 p. 100 pour le douglas, 51 p. 100 pour le hêtre, 15 p. 100 pour le bouleau et 23 p. 100 pour le pin sylvestre.

Nos propres mesures ne permettent pas de trancher cette question car elles n'ont pas été faites dans les mêmes conditions ni à la même échelle de temps. En effet, les mesures faites véritablement in situ pendant de longues périodes font intervenir d'une part les régulations physiologiques propres à l'arbre et d'autre part les réactions de l'arbre aux différents stress extérieurs.

C'est pourquoi il n'est pas possible d'extrapoler des mesures ponctuelles jusqu'au niveau d'une saison tout entière. De plus, la méconnaissance de la physiologie de 
l'arbre dans son ensemble, des différents flux de matière, de la localisation des "puits » et des «sources» ne permet pas d'établir les termes de passage correspondants.

Il est toutefois possible, d'après nos résultats, de mettre en évidence la contribution des différentes parties des houppiers à la production photosynthétique totale du peuplement. Des travaux précédents (Aussenac \& Ducrey, 1977) ont permis de réaliser un profil vertical qui donne l'indice foliaire par tranches horizontales de végétation. Ce profil, combiné avec celui donnant la photosynthèse nette maximale niveau par niveau, permet de déterminer la part de chaque tranche de végétation dans la photosynthèse totale (figure 4).

Cette répartition est évidemment très schématique car elle ne tient compte d'aucun des processus physiologiques qui conditionnent le fonctionnement de chaque partie des houppiers dans un peuplement. On voit que les niveaux II et III, pour lesquels la photosynthèse potentielle est la plus élevée, ne concernent que 11 p. 100 de la surface foliaire de 14 p. 100 de la photosynthèse potentielle. Les niveaux IV et $\mathrm{V}$ qui correspondent à la plus forte zone d'extinction du rayonnement solaire comprennent 62 p. 100 de la surface foliaire totale et 63 p. 100 de la photosynthèse potentielle. Les feuilles franchement à l'ombre et qui reçoivent moins de 15 p. 100 d'éclairement relatif représentent 27 p. 100 de la surface foliaire et participent pour 23 p. 100 à la photosynthèse totale. Ce raisonnement n'est bien entendu valable que si l'éclairement ambiant dans lequel vivent les feuilles est égal ou supérieur à l'éclairement de saturation des courbes photosynthèse nette-éclairement.

Des expérimentations voisines de celles de Schulze \& Koch ou de Künstle \& Mitscherlich, mais faites à de très nombreux niveaux, permettraient daffiner le schéma proposé et de comprendre l'impact des traitements sylvicoles sur le fonctionnement photosynthétique de l'arbre et du peuplement tout entier.

\section{5. - Conclusion}

Les nombreux travaux auxquels il est fait référence tout au long de cet article montrent que le problème de l'adaptation physiologique des organes végétaux poussant à l'ombre ou à la lumière commence à être bien connu. Cependant, au moment de faire une synthèse, d'essayer de dresser des schémas généraux de fonctionnement, on s'aperçoit que les exceptions sont nombreuses.

En effet chaque espèce et, à un degré moindre, chaque arbre ont un comportement physiologique propre qui engendre des variations au niveau du fonctionnement photosynthétique.

C'est pourquoi, si l'on veut progresser dans la connaissance du fonctionnement photosynthétique et de la croissance des arbres, il devient indispensable d'analyser l'arbre dans son ensemble, de rechercher les différents flux de sève brute, de sève élaborée et de substances de croissance, de déterminer l'emplacement des «sources » et des «puits» engendrant ces différents flux et de situer dans l'arbre les zones de plus grande activité physiologique. 


\section{Summary}

\section{Bioclimatological studies in a broad leaf high stand \\ (Fagus silvatica L. and Quercus sessiliflora Salisb.)}

\section{III. - Photosynthetic rates of leaves from various levels in the stand}

Net photosynthesis has been investigated in a broad leaf high stand where some beech and oak twigs have been cut off at ten levels in the tree crowns. The relationships between net photosynthesis and light intensity have been established in a gas-exchange chamber devised for laboratory use and regulated in temperature, air humidity and carbon dioxide concentration.

For beech, maximum net photosynthesis per leaf area unit increases with the light intensity during growth. It increases more than twice from shade leaves $\left(4 \mathrm{mg} \mathrm{CO}_{2} \mathrm{dm}^{-2} \mathrm{~h}^{-1}\right)$ to full light leaves $\left(9 \mathrm{mg} \mathrm{CO} \mathrm{dm}^{-2} \mathrm{~h}^{-1}\right)$. In the same conditions, dry matter per leaf area unit is multiplied by 3.5 . It results that net photosynthesis per dry matter unit decreases as relative daylight of growth increases : it is 50 p. 100 higher in shade leaves than in full light leaves. For oak, similar trends are observed.

These results are related with morphological, anatomical and physiological characteristics of leaves and compared with those from the litterature. Photosynthesis per volume unit of leaf seems to be a constant data, pratically independant of the light intensity during growth and life of the various leaves on a tree.

\section{Références bibliographiques}

Aussenac G., Ducrey M., 1977. Etude bioclimatique dune futaie feuillue (Fagus silvatica L. et Quercus sessiliflora Salisb.) de l'Est de la France. 1. Analyse des profils microclimatiques et des caractéristiques anatomiques et morphologiques de l'appareil foliaire. Ann. Sci. Forest., 34 (4), 265-284.

Aussenac G., Ducrey M., Bouchon J., 1975. La production photosynthétique forestière, 217-242. In : Photosynthèse et production végétale. Costes (Ed.), Gauthier-Villars, Paris.

Aussenac G., Granier A., 1979. Etude bioclimatique d'une futaie feuillue (Fagus silvatica L.) et Quercus sessiliflora Salisb.) de l'Est de la France. 2. Etude de l'humidité du sol et de l'évapotranspiration réelle. Ann. Sci. Forest., 36 (4), 265-280.

Buörkman O., Holmgren P., 1963. Adaptability of the photosynthetic apparatus to light intensity in ecotypes from exposed and shaded habitats. Physiol. Plant., 16, 889-914.

Boardman N.K., 1977. Comparative photosynthesis of sun and shade plants. Ann. Rev. Plant Physiol., 28, 355-377.

DuCRFy M., 1979. Description d'une enceinte régulée en température, humidité et gaz carbonique pour l'étude de la photosynthèse, de la respiration et de la transpiration. Publication Station Sylviculture I.N.R.A.-C.N.R.F., Nancy.

Künstle E.. Mitscherlich G., 1975 (77). Photosynthese, transpiration und atmung in einen Mischbestand in Schwarzwald. I. Teil : Photosynthese. Allg. Forst-u. J. Ztg., 146 (3-4), 45-63. IV. Teil : Bilanz. Allg. Forst-u. J. Ztg., 148 (12), 227-239.

Mousseau M., 1968. Action comparée de la lumière sur l'intensité photosynthétique de feuilles entières coupées ou sur pied, selon les conditions d'éclairement pendant la croissance. C.R. Acad. Sc. Paris. Série D, 266. 1391-1393.

Mousseau M., 1969. Etude écologique de la photosynthèse des formes d'ombre et de lumière de Teucrium Scorodonia L. Analyse de son adaptation aux conditions d'éclairement. Thèse Doct. Sci. Nat. Univ. Paris XI, $123 \mathrm{p}$. 
Moussead M., 1977. Adaptation de la photosynthèse d'une plante tolérante à l'ombrage. Le Teucrium scorodonia, 157-181. In : Les processus de la production végétale primaire. A. Moyse (Ed.), Gauthier-Villars, Paris.

Penka M., Cermak J., Stepanek V., 1971. Contribution to studies of the photosynthetic rate in oak (Quercus robur L.). Acta universitatis agriculturac (Brno). Série C., 40 (4), 283-301.

Schulze E.D., 1970. Der CO... - Gaswechsel der Buche (Fagus silvatica L.) in Abhängigkeit von den Klimafaktoren in Freiland. Flora, 159, 177-232.

Schulze E.D., Koch W., 1971. Measurement of primary production with cuvettes, 141-157. In : Productivity of Forest Ecosystems. Proc. Brussels Symp., Unesco, Paris.

STARzeCKI W., 1974. Topography of photosynthesis in the crowns of Tilia cordata and Acer pseudoplatanus trees. Bull. Acad. Polon. Sci., Ser. Sci. Biol., 22 (9), 597-602.

STARzECKI W., 1975. Ecophysiological investigation on photosynthetic productivity of leaves of chosen tree species. Pol. ecol. stud., 1 (1), 51-63.

Wassink E.C., Richardson S.D., Pieters G.A.. 1956. Photosynthetic adaptation to light intensitv in leaves of Acer pseudoplatanus. Acta Botanica Neerlandica., 5 (3), 247-256. 